\title{
Band-fluctuations model for the fundamental absorption of crystalline and amorphous semiconductors: a dimensionless joint density of states analysis
}

\author{
J. A. Guerra ${ }^{1,2} \dagger$, A. Tejada ${ }^{1,2}$, J. A. Töfflinger ${ }^{1,2}, \mathbf{R}$. \\ Grieseler $^{1}$ and L. Korte ${ }^{2}$ \\ ${ }^{1}$ Departamento de Ciencias, Sección Física, Pontificia Universidad Católica del \\ Perú, Av. Universitaria 1801, Lima 32, Perú \\ ${ }^{2}$ Helmholtz-Zentrum Berlin für Materialien und Energie GmbH, Institut für \\ Silizium-Photovoltaik, Kekuléstraße 5, 12489 Berlin, Germany \\ E-mail: guerra.jorgea@pucp.edu.pe \\ E-mail: korte@helmholtz-berlin.de
}

August 2018

\begin{abstract}
We develop a band-fluctuations model which describes the absorption coefficient in the fundamental absorption region for direct and indirect electronic transitions in disordered semiconductor materials. The model accurately describes both the Urbach tail and absorption edge regions observed in such materials near the mobility edge in a single equation with only three fitting parameters. An asymptotic analysis leads to the universally observed exponential tail below the bandgap energy and to the absorption edge model at zero Kelvin above it, for either direct or indirect electronic transitions. The latter feature allows the discrimination between the absorption edge and absorption tails, thus yielding more accurate bandgap values when fitting optical absorption data. We examine the general character of the model using a dimensionless Joint Density of States formalism with a quantitative analysis of a large amount of optical absorption data. Both heavily doped p-type GaAs and nano-crystalline $\mathrm{Ga}_{1-\mathrm{x}} \mathrm{Mn}_{\mathrm{x}} \mathrm{N}$, as examples for direct bandgap materials, as well as amorphous $\mathrm{Si}: \mathrm{H}_{\mathrm{x}}, \mathrm{SiC}: \mathrm{H}_{\mathrm{x}}$ and $\mathrm{SiN}_{\mathrm{x}}$, are modeled successfully with this approach. We contrast our model with previously reported empirical models, showing in our case a suitable absorption coefficient shape capable of describing various distinct materials while also maintaining the universality of the exponential absorption tail and absorption edge.
\end{abstract}

Submitted to: J. Phys. D: Appl. Phys.

$\dagger$ Corresponding author 


\section{Introduction}

Knowledge of the optical bandgap and Urbach energy of crystalline and amorphous semiconductors is essential when designing materials and devices. For instance, the impact of localized tail states on the performance of optoelectronic devices based on amorphous and/or polycrystalline semiconductors is well established [1-8]. In the vicinity of the absorption edge, electronic transitions between the conduction and valence extended states are responsible for the main optical absorption phenomena in semiconductors, the so-called fundamental band-to-band absorption. This can be characterized using optical data, which contains information on the electronic density of states (DOS) in the bands as well as on the band tail density of states and also within the optical bandgap. To this end, light absorption measurements are one of the most commonly used approaches to estimate the optical bandgap. This is typically done by fitting the fundamental absorption region of the absorption coefficient $\alpha(\lambda)$ with a suitable model. This is a relatively simple and straightforward procedure, however, conventional models used to describe the fundamental absorption region do not consider localized tail states and are in most cases limited to a narrow spectral region. Thus, the retrieved bandgap values are often sensitive to the chosen fitting region and can be unreliable.

Depending on the band structure of a material, there are three models to describe the fundamental absorption: for crystalline direct materials $(\alpha \sim$ $\left.\sqrt{\hbar \omega-E_{\mathrm{g}}}\right)$ and for crystalline indirect materials and amorphous materials $\left(\alpha \sim\left(\hbar \omega-E_{\mathrm{g}}\right)^{2}\right)$. Although the functional dependence on photon energy is the same for indirect and amorphous cases, these are two distinct models by definition [9]. These conventional models are developed for isotropic media in the effective mass approximation at zero Kelvin [9-12]. They do not consider electronic transitions between disorderinduced tail states and extended states. Typically, tail state absorption overlaps with the fundamental absorption, making it difficult to establish a region where the aforementioned models can be used. In amorphous semiconductors the latter effect is more pronounced due to an increased density of disorderinduced tail states.

Exponential absorption tails are typically analyzed using the empirical Urbach rule $\left(\alpha \sim e^{\beta \hbar \omega}\right)[13]$.
This model is independent from the fundamental absorption and does not contain information on the optical bandgap nor is it analytically connected to other physical parameters of the fundamental absorption. In this matter, we have recently proven the effectiveness of introducing band-fluctuations when describing the fundamental absorption of amorphous hydrogenated silicon carbide [1], nano-crystalline methylammonium lead iodide [2] and formamidinium cesium lead mixedhalide [3] perovskite semiconductors near the absorption band edge. Here, we develop the fundamental absorption model in detail, resulting in a merge of the Urbach and absorption edge regions in a single equation described as a consequence of band-fluctuations. An asymptotic analysis leads to an exponential behaviour equivalent to the Urbach rule for photon energies well below the bandgap ( $\hbar \omega \ll E_{\mathrm{g}}$ ), and to the conventional band-to-band extended states absorption models above the absorption edge. This model can therefore be used to distinguish the band-to-band absorption edge from the absorption due to localized-to-extended electronic transitions (absorption tails).

We use this framework to first show that thermally induced band-fluctuations and structural induced band-fluctuations can be treated equivalently. We introduce band-fluctuations in the light-induced electronic transition rate through an averaging of the joint density of states. Then, we expose the general character of the developed fundamental absorption model via a dimensionless joint density of states formalism $[14,15]$, exhibiting the universal nature of the shape of the fundamental absorption which is material independent under the effective mass approximation and contrasting the model with previously developed ones attempting to describe the absorption edge covering the exponential absorption tails. Finally, absorption coefficient data for various direct crystalline and amorphous semiconductors are used to demonstrate the applicability of the developed model. Here we perform the analysis with optical data from crystalline GaAs, nano-crystalline $\mathrm{Ga}_{1-\mathrm{x}} \mathrm{Mn}_{\mathrm{x}} \mathrm{N}$, and amorphous $\mathrm{Si}: \mathrm{H}_{x}, \mathrm{SiN}_{\mathrm{x}}$ and $\mathrm{SiC}: \mathrm{H}_{\mathrm{x}}$.

\section{Theoretical background}

In this section, we briefly review the electronic Joint Density of States (JDOS) concept for direct and indirect materials, which is the prerequisite for our band-fluctuations calculation and dimensionless JDOS 
concept.

The absorption coefficient of a semiconductor is proportional to the energy density rate $\hbar \omega R_{\mathrm{cv}}$, with $\hbar \omega$ the photon energy and $R_{\mathrm{cv}}$ the electronic transition rate $[11,12]$,

$$
\begin{aligned}
R_{\mathrm{cv}}=\mathcal{R} \sum_{k_{\mathrm{c}}, k_{\mathrm{v}}} & \left|M_{\mathrm{cv}}\right|^{2} \\
& \times \delta\left(E_{\mathrm{c}}-E_{\mathrm{v}}-\hbar \omega \pm E_{\Omega}\right) \delta_{k_{\mathrm{c}}, k_{\mathrm{v}}+q \pm k_{\Omega}} .
\end{aligned}
$$

Here, $\mathcal{R}=2 \pi / \hbar\left(\overline{\mathrm{E}} e / 2 \omega m_{e}\right)^{2}$, with $m_{e}$ the electron mass, $e$ the elementary charge and $\overline{\mathrm{E}}$ the electric field of the incoming radiation. $E_{\mathrm{c}}$ and $E_{\mathrm{v}}$ are energies in the conduction and valence energy bands of the initial and final states taking part in the absorption process, respectively. $\left|M_{\mathrm{cv}}\right|^{2}$ is the electronic transition matrix element and is different for indirect and direct electronic transitions. $k_{\mathrm{c}}$ and $k_{\mathrm{v}}$ are the conduction and valence electron wave vectors, respectively. $k_{\Omega}$ and $E_{\Omega}$ are the phonon wave vector and phonon energy, respectively. $q$ is the photon wave vector component $(\omega / c)$. For practical effects $q$ and $E_{\Omega}$ can be neglected. The terms $\delta\left(E_{\mathrm{c}}-E_{\mathrm{v}}-\hbar \omega \pm E_{\Omega}\right)$ and $\delta_{k_{\mathrm{c}}, k_{\mathrm{v}}+q \pm k_{\Omega}}$ ensure the energy and wave vector conservation, respectively.

Note that Eq. (1) represents actually two equations, one for direct and another for indirect electronic transitions. In the first case no inelastic processes are taken into account and thus the most probable absorption events are those that keep $k_{\mathrm{c}}=$ $k_{\mathrm{v}}$, whereas for indirect electronic transitions the transition in $k$-space is phonon assisted and thus $k_{\mathrm{c}}=k_{\mathrm{v}} \pm k_{\Omega}$. In the amorphous case, on the other hand, the $k$-conservation is relaxed allowing all possible transitions [9]. This can be understood in terms of the frozen phonon approach [16].

Typically, in order to develop an analytic model for the fundamental absorption, the energy bands are treated in the effective mass approximation, i.e. $E_{\mathrm{v}}=-\frac{\hbar^{2} k_{\mathrm{v}}^{2}}{2 m_{h}^{*}}$ and $E_{\mathrm{c}}=E_{\mathrm{g}}+\frac{\hbar^{2} k_{\mathrm{c}}^{2}}{2 m_{e}^{*}}$, with $m_{h}^{*}$ and $m_{e}^{*}$ being the effective hole and electron masses, respectively, assumed isotropic. This approximation allows a description of the absorption coefficient in Eq. (1) only within a spectral region near the optical bandgap.

For direct transitions only, $R_{\mathrm{cv}}$ can be written in terms of the JDOS $D_{\mathrm{cv}}$, as shown in Eq. (2). $D_{\mathrm{cv}}$ is given by Eq. (3) and is the result of calculating the density of states of the energy bands difference $E_{\mathrm{cv}}=E_{\mathrm{c}}-E_{\mathrm{v}}$ for $k_{\mathrm{c}}=k_{\mathrm{v}}[11,12]$.

$$
R_{\mathrm{cv}}=\mathcal{R} \int\left|M_{\mathrm{cv}}\right|^{2} D_{\mathrm{cv}}\left(E_{\mathrm{cv}}\right) \delta\left(E_{\mathrm{cv}}-\hbar \omega\right) d E_{\mathrm{cv}}
$$

$$
\begin{aligned}
D_{\mathrm{cv}}\left(E_{\mathrm{cv}}\right)= & \frac{\sqrt{2} \mu^{* 3 / 2}}{\pi^{2} \hbar^{3}} \\
& \times \begin{cases}\left(E_{\mathrm{cv}}-E_{\mathrm{g}}\right)^{1 / 2} & , E_{\mathrm{cv}} \geq E_{\mathrm{g}} \\
0 & , E_{\mathrm{cv}}<E_{\mathrm{g}}\end{cases}
\end{aligned}
$$

Here, $\mu^{*}$ is the effective reduced mass, $\mu^{*-1}=m_{\mathrm{e}}^{*-1}+$ $m_{\mathrm{h}}^{*-1}$. By assuming that $\left|M_{\mathrm{cv}}\right|^{2}$ varies slowly with the energy, the absorption coefficient under these conditions $\left({ }^{d} \alpha\right)$ can then be written as shown in Eq. (4), with $n$ the refractive index and $c$ the speed of light $[11,12]$.

$$
{ }^{d} \alpha(\hbar \omega)=\frac{\pi \hbar}{\epsilon_{0} n c}\left(\frac{e}{m_{e}}\right)^{2} \frac{\left|M_{\mathrm{cv}}\right|^{2}}{\hbar \omega} D_{\mathrm{cv}}(\hbar \omega)
$$

On the other hand, for indirect electronic transitions all energetically possible transitions, for a given photon energy $\hbar \omega$, between initial $(v)$ and final (c) states must be considered in $R_{\mathrm{cv}}$. Thus, $R_{\mathrm{cv}}$ is written in terms of both the valence $\left(D_{\mathrm{v}}\right)$ and conduction $\left(D_{\mathrm{c}}\right)$ DOSs as shown in Eq. (5). This expression is the same for amorphous materials $[9,10]$.

$$
\begin{aligned}
R_{\mathrm{cv}}=\mathcal{R} \iint\left|M_{\mathrm{cv}}\right|^{2} & D_{\mathrm{c}}\left(E_{\mathrm{c}}\right) D_{\mathrm{v}}\left(E_{\mathrm{v}}\right) \\
& \times \delta\left(E_{\mathrm{c}}-E_{\mathrm{v}}-\hbar \omega\right) d E_{\mathrm{v}} d E_{\mathrm{c}}
\end{aligned}
$$

The conduction and valence DOSs, in the effective mass approximation, are given by Eqs. (6) and (7), respectively.

$$
\begin{gathered}
D_{\mathrm{c}}\left(E_{\mathrm{c}}\right)=\frac{\sqrt{2} m_{\mathrm{e}}^{* 3 / 2}}{\pi^{2} \hbar^{3}} \begin{cases}\left(E_{\mathrm{c}}-E_{\mathrm{g}}\right)^{1 / 2} & , E_{\mathrm{c}} \geq E_{\mathrm{g}} \\
0 & , E_{\mathrm{c}}<E_{\mathrm{g}}\end{cases} \\
D_{\mathrm{v}}\left(E_{\mathrm{v}}\right)=\frac{\sqrt{2} m_{\mathrm{h}}^{* 3 / 2}}{\pi^{2} \hbar^{3}} \begin{cases}0 & E_{\mathrm{v}}>0 \\
\left(-E_{\mathrm{v}}\right)^{1 / 2} & , E_{\mathrm{v}} \leq 0\end{cases}
\end{gathered}
$$

Again, by assuming that $\left|M_{\mathrm{cv}}\right|^{2}$ varies slowly with energy, the integral in Eq. (5) has a known solution [12]. It is possible to define a JDOS $J_{\mathrm{cv}}$, in order to write $R_{\mathrm{cv}}$ and the absorption coefficient in a simplified form, such as

$$
R_{\mathrm{cv}}=\mathcal{R} \int\left|M_{\mathrm{cv}}\right|^{2} J_{\mathrm{cv}}\left(E_{\mathrm{cv}}\right) \delta\left(E_{\mathrm{cv}}-\hbar \omega\right) d E_{\mathrm{cv}},
$$

with

$$
\begin{gathered}
J_{\mathrm{cv}}\left(E_{\mathrm{cv}}\right)=\int D_{\mathrm{c}}\left(E_{\mathrm{v}}+E_{\mathrm{cv}}\right) D_{\mathrm{v}}\left(E_{\mathrm{v}}\right) d E_{\mathrm{v}} \\
=\frac{2\left(m_{\mathrm{e}}^{*} m_{\mathrm{h}}^{*}\right)^{3 / 2}}{\pi^{4} \hbar^{6}} \begin{cases}\frac{\pi}{8}\left(E_{\mathrm{cv}}-E_{\mathrm{g}}\right)^{2} & , E_{\mathrm{cv}} \geq E_{\mathrm{g}} \\
0 & , E_{\mathrm{cv}}<E_{\mathrm{g}}\end{cases}
\end{gathered}
$$


The absorption coefficient for indirect transitions $\left({ }^{i} \alpha\right)$ under the above mentioned assumptions is then given by

$$
{ }^{i} \alpha(\hbar \omega)=\frac{\pi \hbar}{\epsilon_{0} n c}\left(\frac{e}{m_{e}}\right)^{2} \frac{\left|M_{\mathrm{cv}}\right|^{2}}{\hbar \omega} J_{\mathrm{cv}}(\hbar \omega) .
$$

Note that Eqs. (4) and (10) are very similar, and the direct or indirect character of the electronic transitions depends on the JDOS.

\subsection{Band-fluctuations}

Band-fluctuations arise from disorder-induced local variations of the band edges, thermally-induced potential fluctuations or lattice vibrations, and any other deviations from the perfect periodicity of the lattice $[17,18]$. In order to introduce band-fluctuations into the optical absorption process, let us first define $Q_{\mathrm{cv}}$ as a general JDOS in the effective mass approximation,

$$
Q_{\mathrm{cv}}\left(E_{\mathrm{cv}} ; E_{\mathrm{g}}\right)=Q_{0}\left\{\begin{array}{ll}
\left(E_{\mathrm{cv}}-E_{\mathrm{g}}\right)^{r} & , E_{\mathrm{cv}} \geq E_{\mathrm{g}} \\
0 & , E_{\mathrm{cv}}<E_{\mathrm{g}}
\end{array} .\right.
$$

Here, for $r=1 / 2: Q_{\mathrm{cv}}=D_{\mathrm{cv}}$ and for $r=2: Q_{\mathrm{cv}}=J_{\mathrm{cv}}$. $Q_{0}$ collects the corresponding coefficients either for direct or indirect electronic transitions. Note that $E_{\mathrm{g}}$ is written explicitly in the argument of $Q_{\mathrm{cv}}$ in order to emphasize the band-fluctuations and the explicit dependency of $Q_{\mathrm{cv}}$ on $E_{\mathrm{g}}$. For this, we consider $Q_{\mathrm{cv}}$ as a local JDOS, with a local bandgap named $z$ which fluctuates around $E_{\mathrm{g}}$ with a distribution $\widehat{W}\left(z-E_{\mathrm{g}}\right)$ of potential fluctuations $[18,19]$,

$$
\left\langle Q_{\mathrm{cv}}\right\rangle\left(E_{\mathrm{cv}} ; E_{\mathrm{g}}\right):=\int Q_{\mathrm{cv}}\left(E_{\mathrm{cv}} ; z\right) \widehat{W}\left(z-E_{\mathrm{g}}\right) d z .
$$

By performing the variable change $\epsilon-E_{\mathrm{g}}=E_{\mathrm{cv}}-$ $z$, and for an even $\widehat{W}$, Eq. (12) leads to

$$
\left\langle Q_{\mathrm{cv}}\right\rangle\left(E_{\mathrm{cv}} ; E_{\mathrm{g}}\right)=\int Q_{\mathrm{cv}}\left(\epsilon ; E_{\mathrm{g}}\right) \widehat{W}\left(\epsilon-E_{\mathrm{cv}}\right) d \epsilon
$$

because the two arguments of $Q_{\mathrm{cv}}$ appear as a difference.

In the latter equation $\epsilon$ is the fluctuating variable. This equation can be interpreted as the average effect of thermal fluctuations on the necessary energy to excite an electron from a valence state to a conduction state. On the time scale of an optical absorption event, displacements due to a thermal distribution of phonons can be considered frozen. Thus this formalism is consistent with the interpretation of the optical absorption fluctuations as resulting from thermal and/or structural fluctuations of the bandgap energy.

We use Eq. (13) to determine the average electronic transition rate $\left\langle R_{\mathrm{cv}}\right\rangle$ for direct and indirect electronic transitions by replacing the corresponding JDOS with its average counterpart in $R_{\mathrm{cv}}$, i.e. $D_{\mathrm{cv}}\left(E_{\mathrm{cv}}\right) \rightarrow\left\langle D_{\mathrm{cv}}\right\rangle\left(E_{\mathrm{cv}}\right) \equiv\left\langle Q_{\mathrm{cv}}\right\rangle\left(E_{\mathrm{cv}} ; E_{\mathrm{g}}\right)$ in Eq. (2) and $J_{\mathrm{cv}}\left(E_{\mathrm{cv}}\right) \rightarrow\left\langle J_{\mathrm{cv}}\right\rangle\left(E_{\mathrm{cv}}\right) \equiv\left\langle Q_{\mathrm{cv}}\right\rangle\left(E_{\mathrm{cv}} ; E_{\mathrm{g}}\right)$ in Eq. (8). When assuming $\left|M_{\mathrm{cv}}\right|^{2}$ as nearly constant versus the photon energy [12], the latter substitution leads to Eqs. (14) and (15) for direct and indirect electronic transitions, respectively.

$$
\begin{array}{r}
\left\langle R_{\mathrm{cv}}\right\rangle=\mathcal{R} \int\left|M_{\mathrm{cv}}\right|^{2} D_{\mathrm{cv}}(\epsilon) \widehat{W}(\epsilon-\hbar \omega) d \epsilon \\
\left\langle R_{\mathrm{cv}}\right\rangle=\mathcal{R} \iint\left|M_{\mathrm{cv}}\right|^{2} D_{\mathrm{c}}\left(E_{\mathrm{v}}+\epsilon\right) D_{\mathrm{v}}\left(E_{\mathrm{v}}\right) \\
\times \widehat{W}(\epsilon-\hbar \omega) d E_{\mathrm{v}} d \epsilon
\end{array}
$$

Note that the integration variable $\epsilon$ is a dummy variable, and therefore Eq. (14) is equivalent to Eq. (2) whilst Eq. (15) is equivalent to Eq. (5), with the exception of the weight function. Furthermore, in the fluctuationless limit, the weight function converges into a Dirac delta function $\widehat{W}(\epsilon-\hbar \omega) \rightarrow \delta(\epsilon-\hbar \omega)$ to fully recover Eqs. (2) and (5).

In both direct and indirect cases, the electronic transition rate $R_{\mathrm{cv}}$ experiences the same variation in the energy conservation term $\delta\left(E_{\mathrm{c}}-E_{\mathrm{v}}-\hbar \omega\right) \rightarrow$ $\widehat{W}\left(E_{\mathrm{c}}-E_{\mathrm{v}}-\hbar \omega\right)$ when introducing band-fluctuations. This variation represents the fluctuations of the energy that are necessary for an optical absorption event to take place.

In principle, the selection of the weight function should aim at describing the underlying statistics behind the fluctuations, which can be either thermal and/or structural. Here, instead of following previous authors who used a Gaussian distribution [17-19], we choose a different distribution which also yields a Dirac delta in the fluctuationless limit.

Consider first the representation of the Dirac delta $\delta(\epsilon)$ as the derivative of the step function $\Theta(\epsilon)$

$$
\delta(\epsilon)=\frac{\partial}{\partial \epsilon} \Theta(\epsilon)=-\frac{\partial}{\partial \epsilon}(1-\Theta(\epsilon)) .
$$

The step function $1-\Theta(\epsilon)$ can be expressed in terms of a sigmoidal function through the limit

$$
1-\Theta(\epsilon)=\lim _{1 / \beta \rightarrow 0} \frac{1}{1+e^{\beta \epsilon}} .
$$

Thus, taking the derivative of the argument of the limit in Eq. (16) we obtain 


$$
\delta(\epsilon)=\lim _{1 / \beta \rightarrow 0} \frac{\beta e^{\beta \epsilon}}{\left(1+e^{\beta \epsilon}\right)^{2}} .
$$

Therefore, we define the weight function $\widehat{W}(\epsilon)$ as

$$
\widehat{W}(\epsilon)=\frac{\beta e^{\beta \epsilon}}{\left(1+e^{\beta \epsilon}\right)^{2}} .
$$

This $\widehat{W}(\epsilon)$ distribution fulfills the normalization condition and has a full width at half maximum (FWHM) equal to $4 \beta^{-1} \operatorname{ArcCosh}(\sqrt{2})$. Also, note that $\widehat{W}(\epsilon)$ is equal to minus the derivative of the Fermi distribution $\left(-\frac{\partial}{\partial \epsilon} f(\epsilon)\right)$, with $\beta$ the Urbach slope, the inverse of the Urbach energy, $E_{\mathrm{U}}=1 / \beta$. Thus, with our choice of weight function we come to an expression similar to the Kubo-Greenwood formula for the electrical conductivity of metals [20], in which stimulated relaxation processes are taken into account for an ensemble of electrons at a finite temperature. In the present case the temperature is fictitious and is a measure of the width of the weight function $\widehat{W}$ describing the fluctuations, and the Fermi level is replaced by a pseudo Fermi level located in the valence band edge $[21,22]$.

Eq. (18) allows writing the integral in Eq. (13) in terms of the Fermi integral $\mathrm{F}_{j}$ and thus of the PolyLogarithm function $\mathrm{Li}_{j+1}$, whose solutions are included in most numerical and data analysis software tools nowadays, see Eq. (19). Here $\Gamma(j+1)$ is the Gamma function of $j+1$.

$$
\begin{aligned}
\mathrm{F}_{j}(x) & =\frac{1}{\Gamma(j+1)} \int_{0}^{\infty} \frac{t^{j}}{\exp (t-x)+1} d t \\
& =-\mathrm{Li}_{j+1}(-\exp (x)) .
\end{aligned}
$$

The averaged JDOS can then be written for direct electronic transitions as

$$
\left\langle D_{\mathrm{cv}}\right\rangle\left(E_{\mathrm{cv}}\right)=-D_{0} \frac{1}{2} \sqrt{\frac{\pi}{\beta}} \operatorname{Li}_{1 / 2}\left(-\mathrm{e}^{\beta\left(E_{\mathrm{cv}}-E_{\mathrm{g}}\right)}\right),
$$

with the following asymptotic behaviour

$$
\left\langle D_{\mathrm{cv}}\right\rangle\left(E_{\mathrm{cv}}\right) \approx D_{0}\left\{\begin{array}{ll}
\left(E_{\mathrm{cv}}-E_{\mathrm{g}}\right)^{1 / 2} & , E_{\mathrm{cv}} \gg E_{\mathrm{g}} \\
\frac{1}{2} \sqrt{\frac{\pi}{\beta}} \mathrm{e}^{\beta\left(E_{\mathrm{cv}}-E_{\mathrm{g}}\right)} & , E_{\mathrm{cv}} \ll E_{\mathrm{g}}
\end{array} .\right.
$$

The averaged JDOS for indirect electronic transitions is given by

$$
\left\langle J_{\mathrm{cv}}\right\rangle\left(E_{\mathrm{cv}}\right)=-J_{0} \frac{1}{4} \frac{\pi}{\beta^{2}} \mathrm{Li}_{2}\left(-\mathrm{e}^{\beta\left(E_{\mathrm{cv}}-E_{\mathrm{g}}\right)}\right)
$$

with the following asymptotic behaviour

$$
\left\langle J_{\mathrm{cv}}\right\rangle\left(E_{\mathrm{cv}}\right) \approx J_{0} \frac{\pi}{8}\left\{\begin{array}{ll}
\left(E_{\mathrm{cv}}-E_{\mathrm{g}}\right)^{2}+\pi / \beta^{2} & , E_{\mathrm{cv}} \gg E_{\mathrm{g}} \\
\frac{2}{\beta^{2}} \mathrm{e}^{\beta\left(E_{\mathrm{cv}}-E_{\mathrm{g}}\right)} & , E_{\mathrm{cv}} \ll E_{\mathrm{g}}
\end{array} .\right.
$$

Here $D_{0}=\frac{\sqrt{2} \mu^{* 3 / 2}}{\pi^{2} \hbar^{3}}$ and $J_{0}=\frac{2\left(m_{\mathrm{e}}^{*} m_{\mathrm{h}}^{*}\right)^{3 / 2}}{\pi^{4} \hbar^{6}}$. Note how both the exponential shape of the Urbach tail and the square root (parabolic) shape of the absorption edge for direct (indirect) electronic transitions are recovered.

\subsection{Alternative models}

Attempts to model the fundamental absorption and/or JDOS near the band edge considering tail states have been made previously. Some approaches aim to merge the universally observed exponential tail smoothly with the absorption edge [14, 15, 23-25]. Others are focused on developing a theory yielding Urbach tails [18, 19, 26-29].

Here, for comparison purposes, we focus on the empirical models by Ullrich $[23,24]$ and O'Leary [14, 15] for crystalline direct bandgap and for amorphous semiconductors, respectively. These models rely on attaching Urbach tail states to extended states under a condition of continuity in the derivative.

In the direct case the JDOS is given by

$$
\tilde{D}_{\mathrm{cv}}\left(E_{\mathrm{cv}}\right)=D_{0}\left\{\begin{array}{ll}
\left(E_{\mathrm{cv}}-E_{\mathrm{g}}\right)^{1 / 2} & , E_{\mathrm{cv}} \geq E_{\mathrm{cv}_{\mathrm{T}}} \\
\frac{1}{\sqrt{2 \beta}} \mathrm{e}^{\beta\left(E_{\mathrm{cv}}-E_{\mathrm{cv}_{\mathrm{T}}}\right)} & , E_{\mathrm{cv}}<E_{\mathrm{cvT}}
\end{array},\right.
$$

with

$$
E_{\mathrm{cv}_{\mathrm{T}}}=E_{\mathrm{g}}+\frac{1}{2 \beta} \text {. }
$$

Here the $E_{\text {cv }_{\mathrm{T}}}$ energy value denotes the point at which the transition from the square root shape to the exponential shape of the curve takes place and is a consequence of the first derivative continuity condition $[23,24]$.

Figure 1 depicts the normalized JDOS for direct transitions calculated using the band-fluctuations approach $\left\langle D_{\text {cv }}\right\rangle$ and Ullrich's empirical model $\tilde{D}_{\mathrm{cv}}$. In the band-fluctuations case, we observe how the number of states above the bandgap is reduced and the shape of the JDOS changes by increasing the Urbach energy, whilst tail states increase.

In the indirect (disordered) case, tail states are attached to the valence density of states $\left(D_{\mathrm{v}}\right)$ only, arguing that the conduction band tail states are considerably narrower than the valence band tail states, and thus can be neglected for instance in the particular case of a-Si:H $[14,15,30]$, 


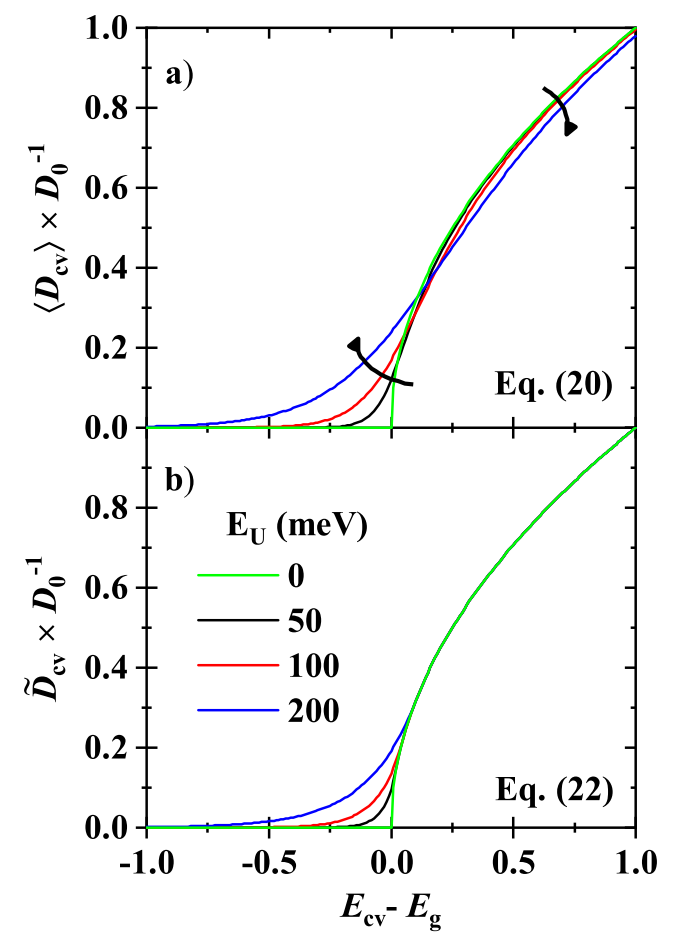

Figure 1. Normalized JDOS curves for direct electronic transitions according to the band-fluctuations model, Eq. (20) $\left\langle D_{\text {cv }}\right\rangle_{\tilde{D}}$ (a) and according to the empirical model by Ullrich, Eq. (22) $\tilde{D}_{\text {cv }}(\mathrm{b})$. Note how the band-fluctuations not only generate tail states below the bandgap in $\left\langle D_{\mathrm{cv}}\right\rangle$ but reduce the number of states above it.

$$
\tilde{D}_{\mathrm{v}}\left(E_{\mathrm{v}}\right)=\frac{\sqrt{2} m_{\mathrm{h}}^{* 3 / 2}}{\pi^{2} \hbar^{3}} \begin{cases}\frac{1}{\sqrt{2 \beta_{\mathrm{v}}}} \mathrm{e}^{\beta_{\mathrm{v}}\left(E_{\mathrm{v}}-E_{\mathrm{v}}\right)} & , E_{\mathrm{v}}>E_{\mathrm{v}_{\mathrm{T}}} \\ \left(-E_{\mathrm{v}}\right)^{1 / 2} & , E_{\mathrm{v}} \leq E_{\mathrm{vT}}\end{cases}
$$

with

$$
E_{\mathrm{v}_{\mathrm{T}}}=-\frac{1}{2 \beta_{\mathrm{v}}} .
$$

Here, also the $E_{\mathrm{v}_{\mathrm{T}}}$ energy value denotes the merging point between the exponential tail and the square root shape of the valence DOS. The JDOS can then be calculated by solving the integral

$$
\tilde{J}_{\mathrm{cv}}(\hbar \omega)=\int D_{\mathrm{c}}\left(E_{\mathrm{v}}+\hbar \omega\right) \tilde{D}_{\mathrm{v}}\left(E_{\mathrm{v}}\right) d E_{\mathrm{v}}
$$

The previous approach can be extended beyond a-Si:H to other amorphous semiconductors by considering the fact that conduction and valence tail states contribute equally to the absorption tail and thus are indistinguishable after the convolution denoted by the integral in Eq. (26). The solution of this integral will be shown in the next section.

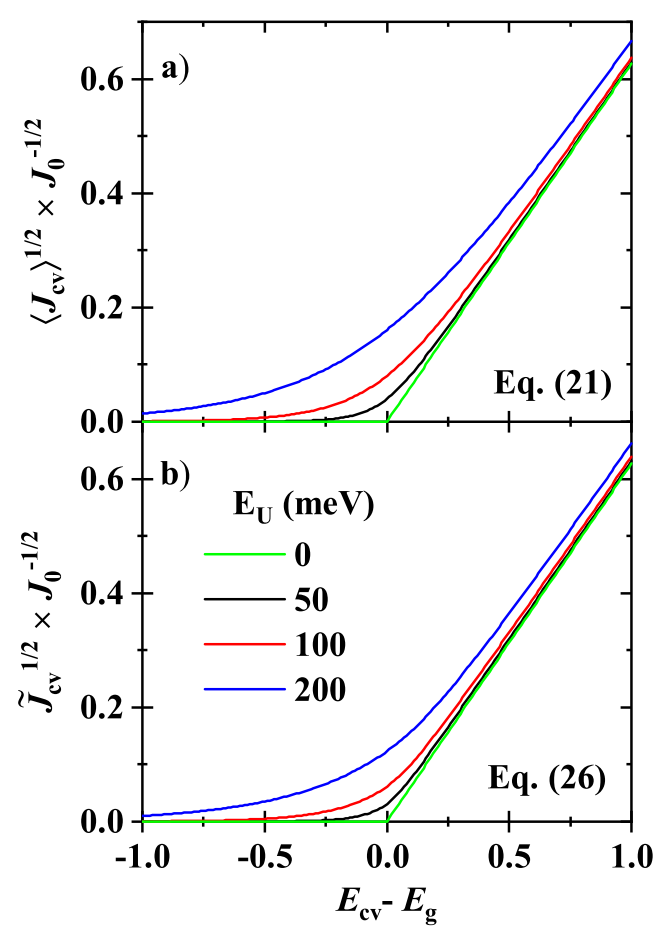

Figure 2. Normalized JDOS curves for indirect electronic transitions according to the band-fluctuations model, Eq. (21) $\left\langle J_{\mathrm{cv}}\right\rangle$ (a) and according to the empirical model by O'Leary, Eq. (26) $\tilde{J}_{\mathrm{cv}}$ (b). The curves are plotted in the square root scale for visualization reasons only, exhibiting the Tauc and Urbach regions merged.

Figure 2 depicts the normalized JDOS for indirect transitions calculated using the band-fluctuations approach $\left\langle J_{\mathrm{cv}}\right\rangle$ and the empirical model by O'Leary $\tilde{J}_{\mathrm{cv}}$. Since in this case the JDOS exhibits a parabolic behaviour, the curves are shown in the square root (or Tauc [10]) scale. Both are very similar and the differences will stand out in the dimensionless JDOS framework described in the next section.

\section{Dimensionless JDOS formalism}

The main achievements of a band-fluctuation model are twofold: (1) the ability to perform a single fit of both tail and extended states with just three parameters, the bandgap $E_{\mathrm{g}}$, Urbach slope $\beta$ and prefactor $\alpha_{0}$, thus extending the fit from the absorption edge region to the tail region, including their overlap [1-3]. This overlap is smooth and follows from a potential fluctuation distribution defined by $\widehat{W}(\epsilon)$. (2) the capability of discriminating the absorption edge of extended-to-extended states transitions from tail states absorption [1-3]. This feature was demonstrated previously for the case of a-SiC:H [1], in which the decrease of the bandgap calculated with the 
Tauc model was attributed to a variation of the Urbach slope, which overlapped considerably with the fundamental absorption edge (or extended states edge). In fact, the bandgap calculated with the bandfluctuations model exhibited a behaviour that was independent of the Urbach energy [1].

A dimensionless JDOS formalism establishes a framework in which experimental results, even from different materials, can be re-scaled onto a single universal curve for comparison purposes [14,15].

In this section we proceed by rewriting the aforementioned models in a dimensionless formalism of a universal shape in the absorption spectrum, which can be traced back to the corresponding JDOS. Such universal shapes are not new. In fact, in the effective mass approximation a square-root (parabolic) shape for the absorption edge is expected, and therefore a similar behaviour between distinct direct (indirect) bandgap materials is also expected. Similar arguments hold for the universality of the exponential tails. As mentioned previously, the general consensus is that Urbach tails are caused by lattice static disorder as well as thermal effects [31,32].

By defining

$$
\mathcal{D}_{\text {cv }}(z)=-\frac{\sqrt{\pi}}{2} \operatorname{Li}_{1 / 2}\left(-\mathrm{e}^{z}\right),
$$

and

$$
\tilde{\mathcal{D}}_{\text {cv }}(z)= \begin{cases}\frac{1}{\sqrt{2}} \mathrm{e}^{\left(z-\frac{1}{2}\right)} & , z<\frac{1}{2} \\ \sqrt{z} & , z \geq \frac{1}{2}\end{cases}
$$

we can write the $D_{\mathrm{cv}}$ in terms of the dimensionless JDOS $\mathcal{D}_{\mathrm{cv}}$ or $\tilde{\mathcal{D}}_{\mathrm{cv}}$ for band-fluctuations or Ullrich's empirical model, respectively,

$$
D_{\mathrm{cv}}\left(E_{\mathrm{cv}}\right)=D_{0} \frac{1}{\sqrt{\beta}} \mathcal{D}_{\mathrm{cv}}\left[\beta\left(E_{\mathrm{cv}}-E_{\mathrm{g}}\right)\right]
$$

Likewise, by defining

$$
\mathcal{J}_{\mathrm{cv}}(z)=-\frac{\pi}{4} \operatorname{Li}_{2}\left(-\mathrm{e}^{z}\right)
$$

and after integrating Eq. (26)

$$
\begin{aligned}
& \tilde{\mathcal{J}}_{\mathrm{cv}}(z)= \\
& \begin{cases}z^{2} \Xi\left[\frac{z-1 / 2}{z}\right]+\frac{1}{\sqrt{2}} \mathrm{e}^{\left(z-\frac{1}{2}\right)} \mathcal{Y}\left[z-\frac{1}{2}\right] & , z \geq \frac{1}{2} \\
\frac{1}{\sqrt{2}} \mathrm{e}^{\left(z-\frac{1}{2}\right)} \mathcal{Y}[0] & , z<\frac{1}{2}\end{cases}
\end{aligned}
$$

with

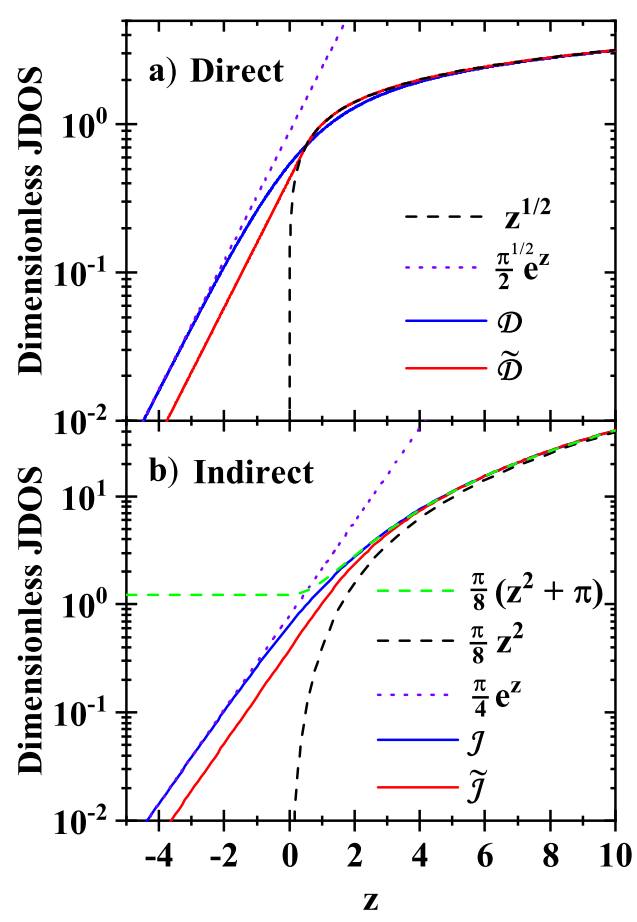

Figure 3. Dimensionless JDOS, Eqs. (27) and (28) for direct (a) and Eqs. (30) and (31) for indirect (and amorphous) (b) electronic transitions. Dashed and dotted lines correspond to the asymptotic limits. Note that the expressions $z^{1 / 2}$ and $z^{2}$ are only valid for $z \geq 0$.

$$
\begin{aligned}
\Xi(z)= & \int_{0}^{z} \sqrt{x} \sqrt{1-x} d x \\
= & \frac{\pi}{8}+\frac{\sqrt{z-z^{2}}}{4}(2 z-1) \\
& \quad+\frac{\sqrt{z-1}}{4} \frac{\sinh ^{-1}(\sqrt{z-1})}{\sqrt{1-z}}
\end{aligned}
$$

and

$$
\begin{aligned}
\mathcal{Y}(z) & =\int_{z}^{\infty} \sqrt{x} \mathrm{e}^{-x} d x \\
& =\sqrt{z} \mathrm{e}^{-z}+\frac{\sqrt{\pi}}{2} \operatorname{erfc}(\sqrt{z})
\end{aligned}
$$

we can write the $J_{\mathrm{cv}}$ in terms of the dimensionless JDOS $\mathcal{J}_{\text {cv }}$ or $\tilde{\mathcal{J}}_{\mathrm{cv}}$ for band-fluctuations, or the empirical model by O'Leary, respectively,

$$
J_{\mathrm{cv}}\left(E_{\mathrm{cv}}\right)=J_{0} \frac{1}{\beta^{2}} \mathcal{J}_{\mathrm{cv}}\left[\beta\left(E_{\mathrm{cv}}-E_{\mathrm{g}}\right)\right] .
$$

Equations (29) and (34) establish a direct relation between the absorption coefficient and the 
corresponding dimensionless JDOS when $z=\beta(\hbar \omega-$ $\left.E_{\mathrm{g}}\right)$. The absorption coefficient can then be written as

$$
{ }^{d} \alpha(\hbar \omega)=\frac{\alpha_{0}}{\hbar \omega} \frac{1}{\sqrt{\beta}} \mathcal{D}_{\mathrm{cv}}\left(\beta\left(\hbar \omega-E_{\mathrm{g}}\right)\right)
$$

for direct transitions, and

$$
{ }^{i} \alpha(\hbar \omega)=\frac{\alpha_{0}}{\hbar \omega} \frac{1}{\beta^{2}} \mathcal{J}_{\mathrm{cv}}\left(\beta\left(\hbar \omega-E_{\mathrm{g}}\right)\right)
$$

for indirect transitions. Here the coefficient $\alpha_{0}$ collects the corresponding constants in a single coefficient either for direct or indirect electronic transitions.

Fig. 3 depicts the dimensionless JDOSs $\mathcal{D}_{\mathrm{cv}}, \tilde{\mathcal{D}}_{\mathrm{cv}}$, $\mathcal{J}_{\text {cv }}$ and $\tilde{\mathcal{J}}_{\mathrm{cv}}$. Additionally, the asymptotic limits are also shown as colored dashed lines. Note that in both direct and indirect cases, Urbach tails have the same slope in logarithmic scale and are independent of the model. Dashed black lines depict the fluctuationless limit in this figure.

\section{Comparison with experiments}

In this section we use the previously developed and described models to analyse the absorption coefficient of distinct materials. We apply the models to two groups of materials: (1) crystalline direct semiconductors exhibiting considerable Urbach tails induced by doping and nano-crystalline structure and (2) disordered semiconductors in which the Urbach tail and mobility edge are modified by hydrogen dilution during the deposition process, thermally induced hydrogen outdiffusion and/or structural relaxation.

For the case of direct semiconductors, we use the absorption coefficient measured for heavily p-doped c-GaAs as shown by Casey et al. [33] and of nc$\mathrm{Ga}_{1-x} \mathrm{Mn}_{x} \mathrm{~N}$ provided by Leite and Da Silva [34]. For the amorphous case we took the absorption coefficient data of a-Si: $\mathrm{H}_{x}$ for different hydrogen dilution contents, extracted from Viturro et al. [35]. We also test the model on the absorption coefficient of a- $\mathrm{SiN}_{x}$ grown in our labs by PECVD [36]. Finally, we present fits for the absorption coefficient of a-SiC: $\mathrm{H}_{x}$ for different hydrogen dilution conditions and after annealing at different temperatures. Further details on the latter samples, such as preparation and optical measurements can be found in Guerra et al. [1].

\subsection{Direct semiconductors}

Doping a crystalline semiconductor induces disorder in the host matrix. Disorder-induced localized states will become evident in the Urbach tail of the absorption coefficient below the bandgap. In particular, heavily doped crystals exhibit considerable tail states, mainly caused by band-fluctuations due to charged impurities randomly distributed in the solid [37]. This is the
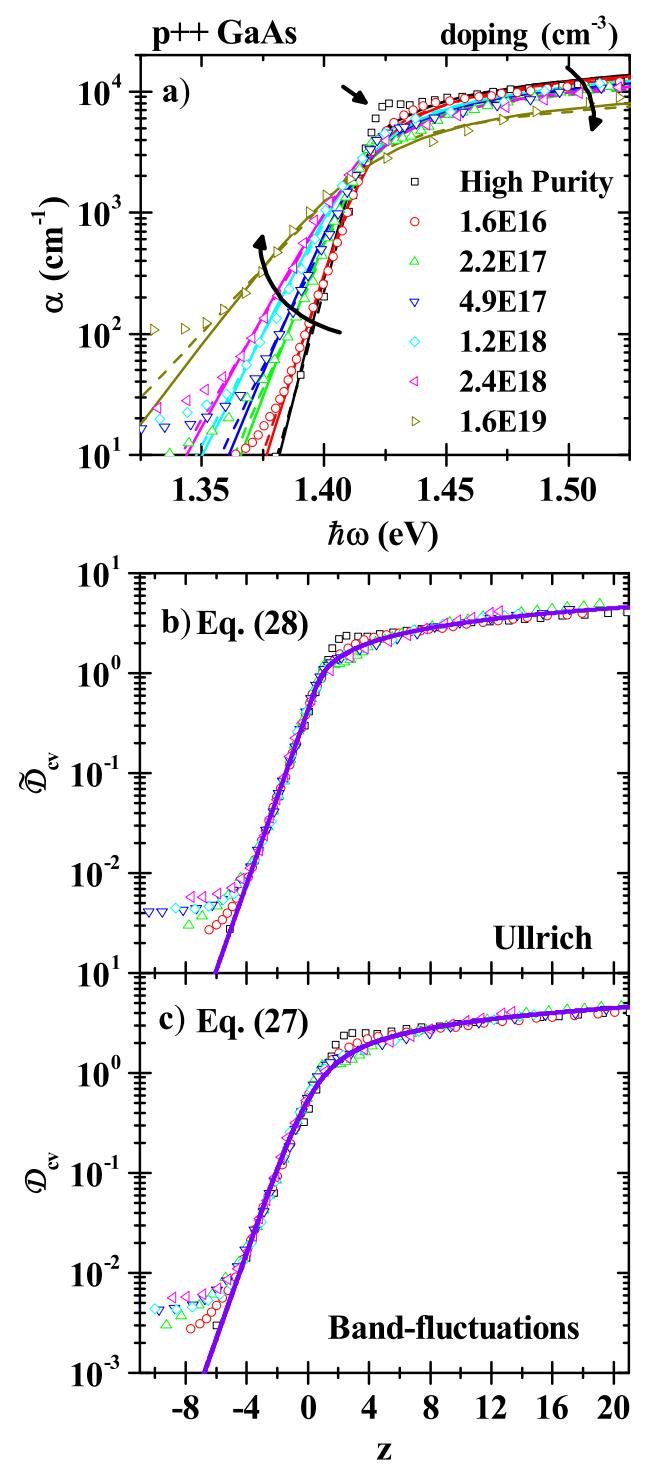

Figure 4. Absorption coefficient of heavily p-doped c-GaAs for different doping concentrations measured at room temperature [33] and the corresponding fits using the models by Ullrich and band-fluctuations, Eqs. (22) and (20), dashed and solid curves, respectively (a). Dimensionless JDOS according to the model by Ullrich $[23,24]\left(\tilde{\mathcal{D}}_{c v}\right)$ described by Eq. (28) (b), and according to the band-fluctuations $[2,3]$ model $\left(\mathcal{D}_{c v}\right)$ described by Eq. (27) (c). The model curve is denoted by a solid purple line. The straight arrow indicates the presence of excitonic states in the high purity sample which were not considered in the model.

case for heavily doped GaAs [33]. Figure 4 depicts fits using Eq. (4) for the absorption coefficient of pdoped c-GaAs for different doping conditions. The fits are performed for the band-fluctuations model and the empirical model by Ullrich, Eqs. (20) and (22), respectively. The corresponding dimensionless JDOSs, using both previously described models for crystalline direct semiconductors $\mathcal{D}_{c v}$ and $\tilde{\mathcal{D}}_{c v}$ are also shown in this figure. The fits achieved with both equations are 

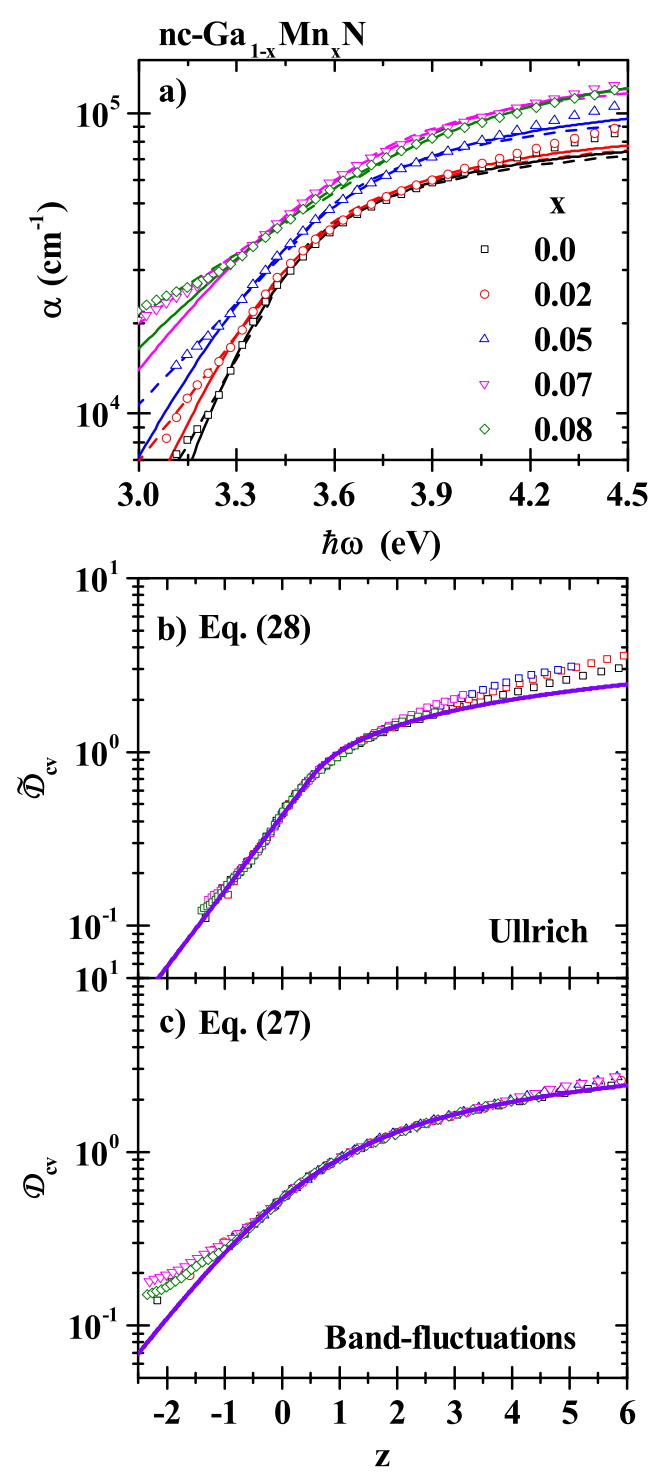

Figure 5. Absorption coefficient of nc- $\mathrm{Ga}_{1-\mathrm{x}} \mathrm{Mn}_{\mathrm{x}} \mathrm{N}$ for different stochiometries measured at room temperature [34] and the corresponding fits using the models by Ullrich and bandfluctuations, Eqs. (22) and (20), dashed and solid curves, respectively (a). Dimensionless JDOS according to the model by Ullrich $[23,24]\left(\tilde{\mathcal{D}}_{c v}\right)$ described by Eq. (28) (b), and according to the band-fluctuations model $[2,3]\left(\mathcal{D}_{c v}\right)$ described by Eq. (27) (c). The model curve is denoted by a solid purple line. Note the poor agreement between experiment and $\tilde{\mathcal{D}}_{c v}$.

good. However, note how in the experimental data the increase of the Urbach tails is followed by a decrease of absorption above the bandgap when increasing the doping content. This behaviour is similar to the one described by $D_{\text {cv }}$ in Eq. (20) and is also depicted in Fig. 1. On the contrary, the empirical model by Ullrich $\tilde{D}_{\text {cv }}$ in Eq. (22) does not reproduce this behaviour.

Nano-crystalline semiconductors also exhibit wide Urbach tails which can be traced back to residual stress after the growth process and/or to different grain sizes.

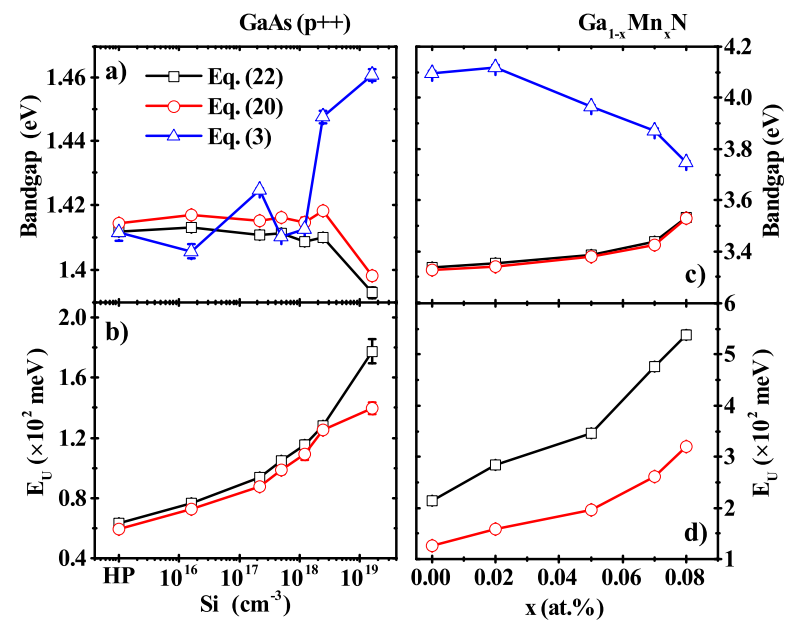

Figure 6. Optical bandgap and Urbach energy values retrieved from the absorption coefficient data of c-GaAs [33] versus $\mathrm{Si}$ doping concentration (a)(b), and $n c-\mathrm{Ga}_{1-\mathrm{x}} \mathrm{Mn}_{\mathrm{x}} \mathrm{N}$ [34] versus stoichiometry $(\mathrm{c})(\mathrm{d})$. The values were obtained using the traditional fundamental absorption model Eq. (3), bandfluctuations model Eq. (20) and Ullrich's model Eq. (22). HP stands for high purity.

Morever, there is a relation that has been observed in some compounds between the stoichiometry of the compound and the width of tail states [38, 39]. Typically, an increase of strained bonds due to a variation in the stoichiometry is followed by an increase of the Urbach energy. Here we fit the absorption coefficient data of nc- $\mathrm{Ga}_{1-x} \mathrm{Mn}_{x} \mathrm{~N}$ obtained from Leite et al. [34]. Fits using Eqs. (20) and (22) are depicted in Fig. 5, along with the corresponding data in the dimensionless JDOS frame. Fits using both models are good. Nevertheless, note how the sharper transition from the extended states region to the tails states region when using Eq. (22) results in a poorer fit in contrast to the band-fluctuations model. This effect becomes more evident in the dimensionless JDOS framework.

The differences between model and experimental data when using the model by Ullrich, i.e. Eq. (22), especially in the overlap of the extended and localized tail states, can be traced back to the arbitrary and/or non-physical merging condition between Urbach tail states and extended states. The continuity of the first derivative seems not to be a suitable condition to model this region. Fig. 6 summarizes the recovered Urbach energy and optical bandgap from the fits shown in Figs. 4 and 5. Additionally, the bandgap values calculated using the fluctuationless JDOS of Eq. (3) are presented for comparison purposes.

On the one hand, for GaAs the retrieved bandgap values match very well between models with an $E_{\mathrm{g}} \approx 1.41 \mathrm{eV}$ for the high purity case. This value in particular is very close to that reported 
by Ullrich et al. [40] at room temperature from photo-luminescence measurements. The behaviour of the bandgap versus the silicon doping follows a similar trend with increasing doping concentration after fitting the band-fluctuations model Eq. (20) and Ullrich's model Eq. (22), in contrast to the values retrieved using the fluctuationless model Eq. (3), which considers only extended-to-extended band-band transitions. The increase of the Urbach energy in this case is associated to band-fluctuations due to the increase of charged impurities [37], whilst the decrease of the bandgap at high doping concentrations is associated to a bandgap narrowing due to many-body effects which become important at shorter carrier-tocarrier distances [37], see Fig. 6 (a) and (b).

On the other hand, for $n c-\mathrm{Ga}_{1-\mathrm{x}} \mathrm{Mn}_{\mathrm{x}} \mathrm{N}$ the bandgap values obtained after fitting Eqs. (20) and (22) are consistent for $x=0\left(E_{\mathrm{g}} \approx 3.35 \mathrm{eV}\right)$. However, in this case the bandgap retrieved using the fluctuationless fundamental absorption model of Eq. (3) shows inconsistent values above $3.4 \mathrm{eV}$ for all compositions. Moreover, the dependence of the bandgap versus the composition $x$ seems to follow inversely the $E_{\mathrm{U}}$ behaviour, see Fig. 6 (c) and (d). This is mainly due to the fact that Eq. (3) does not consider tail states and thus the apparent shrinking of $E_{\mathrm{g}}$ is actually due to the increasing overlap of the Urbach tail on the fundamental absorption edge with the composition [1].

\subsection{Amorphous semiconductors}

In amorphous semiconductors, absorption from tail states to extended states overlaps considerably with the band-to-band absorption edge. Also, similar to the crystalline case, tail states are sensitive to doping, temperature and disorder. The latter in particular can be modified through thermal annealing treatments, thus affecting the Urbach slope directly. In order to evaluate the models developed in previous paragraphs, in this section we test them for three distinct cases: (1) the modification of the fundamental absorption due to variation of hydrogen dilution conditions during the deposition process of a-Si:H, (2) bandgap engineering by means of stochiometry manipulation in a-SiN $\mathrm{S}_{\mathrm{x}}$ and (3) the effect of hydrogen dilution and post deposition thermal annealing treatments on the Urbach tail and absorption edge of a-SiC: $\mathrm{H}_{\mathrm{x}}$.

Fig. 7 depicts the absorption coefficient of aSi:H grown with different hydrogen dilutions during the deposition process. The data were extracted from Vitturo et al. [35]. Fits using Eq. (10) for bandfluctuations and the empirical model by O'Leary, Eqs. (21) and (26), respectively, are also shown in this figure. Both models exhibit a good correspondence between experimental data and theoretical curves.
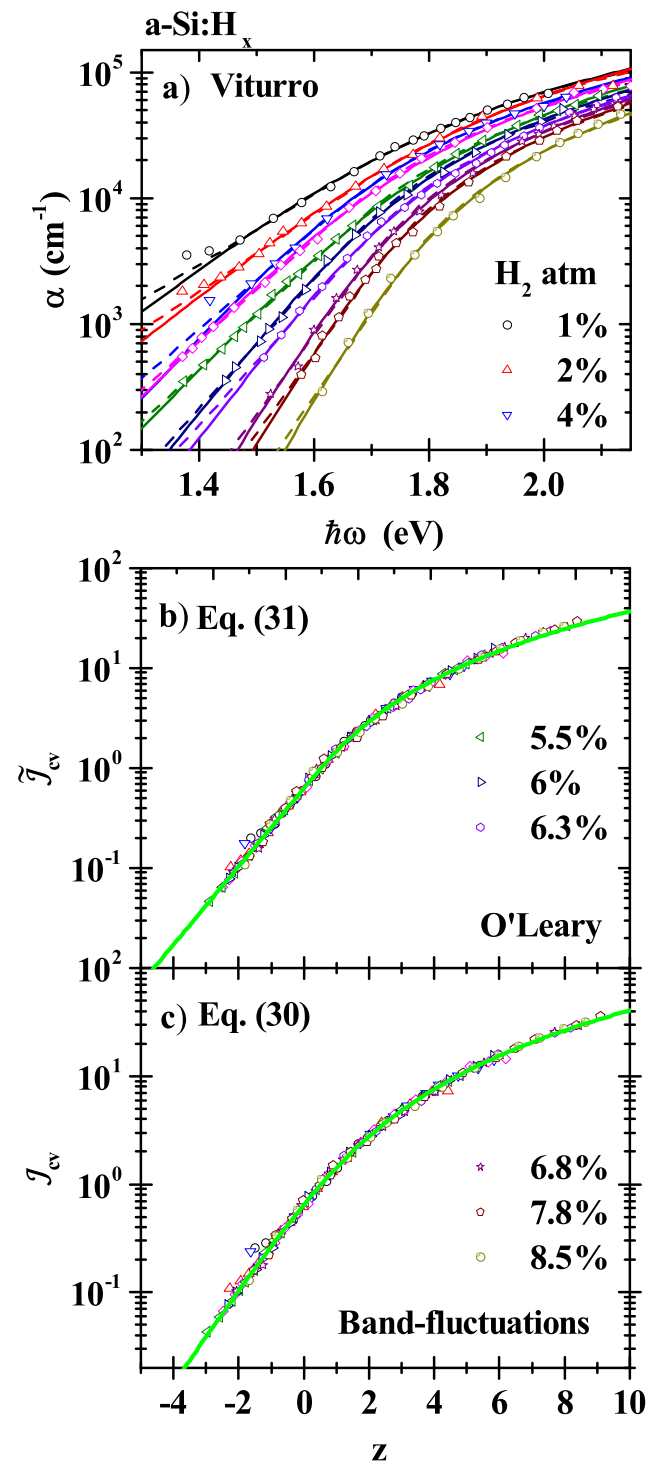

Figure 7. Absorption coefficient of a-Si:H for different hydrogen dilution conditions, measured at room temperature [35] and the corresponding fits using Eqs. (21) and (26), solid and dashed curves, respectively (a). Dimensionless JDOS according to the model by O'Leary [15] $\left(\tilde{\mathcal{J}}_{c v}\right)$ described by Eq. (31) (b), and according to the band-fluctuations $[1]$ model $\left(\mathcal{J}_{c v}\right)$ described by Eq. (30) (c). The model curve is denoted by a solid green line.

Furthermore, no considerable deviation is observed in the dimensionless JDOS frame. The same behaviour is observed for the case of $\mathrm{a}-\mathrm{SiN}_{\mathrm{x}}$ and $\mathrm{a}-\mathrm{SiC}: \mathrm{H}_{\mathrm{x}}$, see Figures 8 and 9 , respectively. Despite the variation of the Urbach slope, a shift of $E_{\mathrm{g}}$ to higher energies is observed following the mobility edges widening due to increasing nitrogen (hydrogen) $[1,8,31]$ incorporation.

Fig. 10 summarizes the recovered Urbach energy and optical bandgap calculated from the fits shown in Figures 8 and 9 for amorphous $\mathrm{SiN}_{\mathrm{x}}$ and $\mathrm{SiC}: \mathrm{H}_{\mathrm{x}}$, respectively. Here only the results corresponding to the $\mathrm{SiC}: \mathrm{H}_{\mathrm{x}}$ samples grown in an argon/hydrogen 

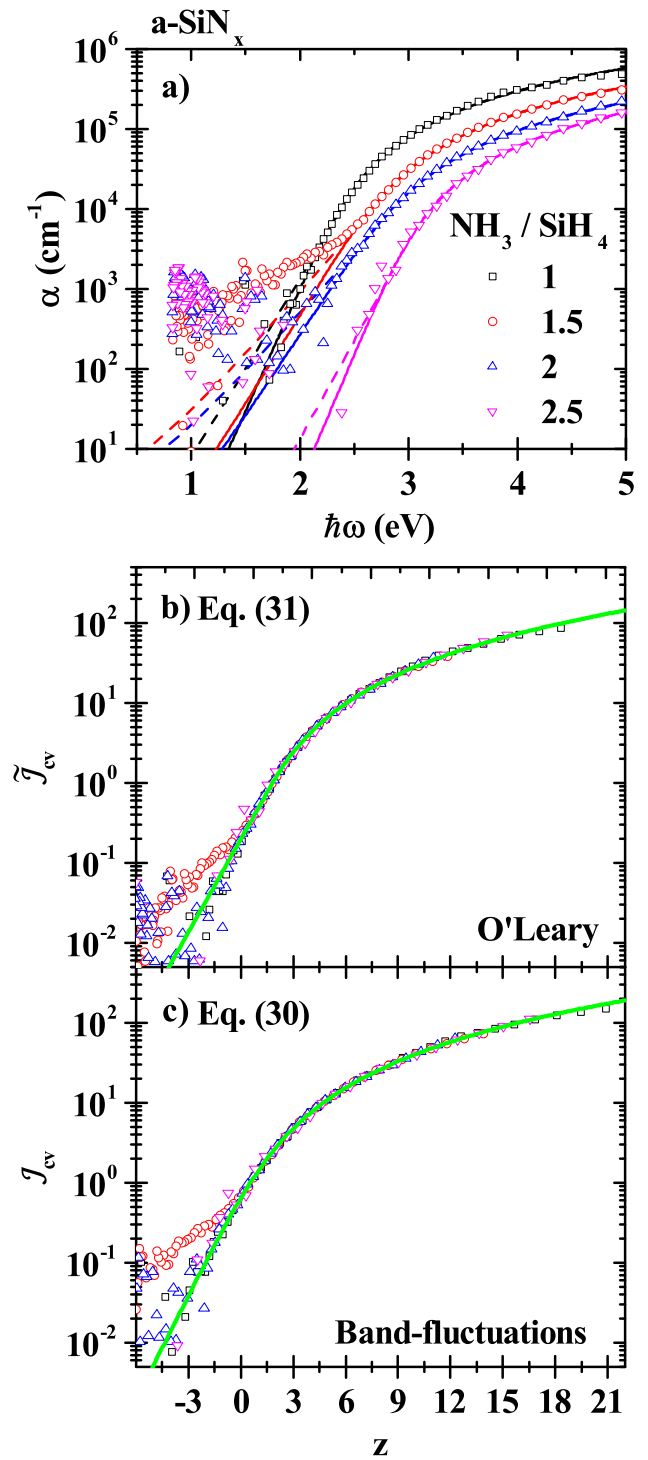

Figure 8. Absorption coefficient of a-SiN ${ }_{x}$ grown with different $\mathrm{NH}_{3} / \mathrm{SiH}_{4}$ flow ratios during the deposition process, measured at room temperature, and the corresponding fits using Eqs. (21) and (26), solid and dashed curves, respectively (a). Dimensionless JDOS according to the model by O'Leary [15] $\left(\tilde{\mathcal{J}}_{c v}\right)$ described by Eq. (31) (b), and according to the bandfluctuations [1] model $\left(\mathcal{J}_{c v}\right)$ described by Eq. (30) (c). The model curve is denoted by a solid green line.

atmosphere with a $15 \mathrm{sccm}$ hydrogen flux are shown for different annealing temperatures. Bandgap values calculated using the fluctuationless JDOS in Eq. (9) are also presented for comparison purposes. The retrieved bandgap values match very well between models for the case of $\mathrm{SiN}_{\mathrm{x}}$ and with the same trend versus the nitrogen incorporation.

On the contrary, bandgap values retrieved with the three different models in the case of a-SiC: $\mathrm{H}_{\mathrm{x}}$ are different and follow different trends when plotted versus the annealing temperature. The bandgap
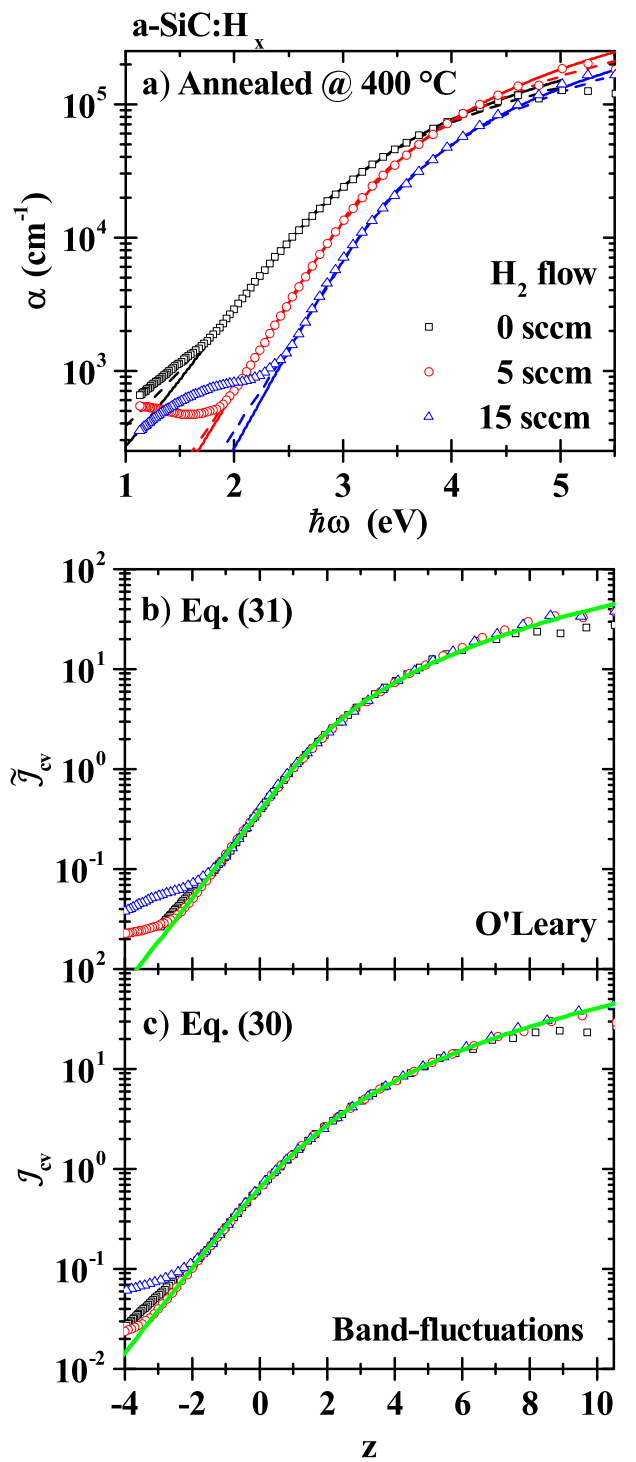

Figure 9. Absorption coefficient of a-SiC: $\mathrm{H}_{\mathrm{x}}$ grown under different hydrogen flows during the deposition process, measured at room temperature [1]. Fits using the band-fluctuations model Eq. (21) and model by O'Leary Eq. (26) are shown, solid and dashed curves, respectively (a). Dimensionless JDOS according to the model by O'Leary [15] $\left(\tilde{\mathcal{J}}_{c v}\right)$ described by Eq. (31) (b), and according to the band-fluctuations model $\left(\mathcal{J}_{c v}\right)$ described by Eq. (30) (c). The model curve is denoted by a solid green line.

calculated with the band-fluctuations model increases with the annealing temperature, while that calculated with the models by O'Leary and Tauc seems to be correlated to the Urbach energy. The expected behaviour with the annealing is a shrinking of the mean lattice constant and thus an enhancing of the bandgap independently of thermally-induced hydrogen out-diffusion or even for non hydrogenated samples [1]. 


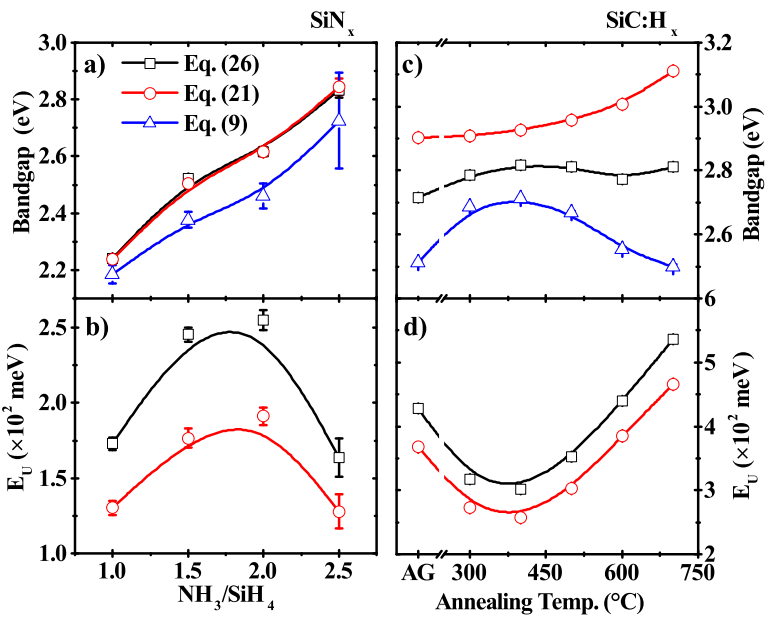

Figure 10. Optical bandgap and Urbach energy retrieved from the absorption coefficient data of a-SiN $\mathrm{N}_{x}$ grown with different $\mathrm{NH}_{3} / \mathrm{SiH}_{4}$ ratios (a)(b), and of a-SiC: $\mathrm{H}_{\mathrm{x}}$ grown with a 15 sccm hydrogen flux during the deposition process for different annealing temperatures (c)(d). The values were obtained using the Tauc model Eq. (9), band-fluctuations model Eq. (21) and model by O'Leary Eq. (26).

\section{Conclusions}

We have developed analytic expressions based on a band-fluctuations model, which are capable of describing the absorption coefficient in the Urbach tail and absorption edge regions of disordered semiconductors with three fitting parameters only. These expressions were developed for the cases of both direct and indirect electronic transitions. We tested these models on absorption data from various materials exhibiting considerable Urbach tails, for instance for direct materials with heavy doping (GaAs), nano crystalline structure and distinct stochiometries $\left(\mathrm{Ga}_{1-\mathrm{x}} \mathrm{Mn}_{\mathrm{x}} \mathrm{N}\right)$. We also tested the model for indirect electronic transitions on amorphous semiconductors, where Urbach tails and mobility edges are modified due to changing hydrogen content in the film, annealing temperature (a-Si:H and a-SiC:H) or stochiometry (a$\mathrm{SiN}_{\mathrm{x}}$ ). We find that our band-fluctuations model provides a suitable description of the overlap between the Urbach and absorption edge regions without introducing new additional states.

An asymptotic analysis of our developed model leads to the universally observed exponential absorption tail for direct and indirect electronic transitions [9]. Moreover, the universality of the effective mass approximation is also recovered when performing the dimensionless JDOS analysis. For photon energies above the bandgap energy, our model reproduces the $\alpha(\hbar \omega)$ behaviour that follows from the effective mass approximation, i.e. parabolic bands lead to a square root shape of the electronic density of states. Using the mentioned examples, we could demonstrate that our models are of great utility to the experimentalist. For instance, by using these models, the band-to-band absorption edge can be distinguished from absorption due to localized-to-extended electronic transitions (tails), and the fitting region for obtaining the optical bandgap can be extended [1-3], yielding a more reliable calculation of the bandgap. Furthermore, the generalized framework can be used to test different distribution functions to describe band-fluctuations, provided they can be cast into an expression describing the energetic distribution of states.

\section{Acknowledgments}

This work was funded by the Research Management Office of the Pontificia Universidad Católica del Perú (DGI-PUCP) and the Peruvian National Fund for Scientific and Technological Development (FONDECYT), grant no. 147-2017. Additional support has been provided by the German Academic Exchange Service (DAAD) and FONDECYT, grant no. 037-2016. The authors are indebted to Martina Trahms (HZB) for the deposition of the $\operatorname{SiN}_{\mathrm{x}}$ samples, and to Fernando Da Silva and Douglas Leite (UNESP) for providing the $\mathrm{Ga}_{1-\mathrm{x}} \mathrm{Mn}_{\mathrm{x}} \mathrm{N}$ absorption coefficient data.

\section{References}

[1] J. A. Guerra et al., J. Phys. D: Appl. Phys. 49(19), 195102 (2016).

[2] J. A. Guerra et al., J. Appl. Phys. 121(17), 173104 (2017).

[3] A. Tejada et al., J. Appl. Phys. 123(17), 175302 (2018).

[4] R. Vasudevan et al., Solar Energy Materials \& Solar Cells 150(3), 92 (2016).

[5] R. Asadpour et al., Appl. Phys. Lett. 106(24), 243902 (2015).

[6] W. E. I. Sha et al., Appl. Phys. Lett. 106(22), 21104 (2015).

[7] S. Schonau et al., Appl. Phys. Lett. 103(19), 192108 (2013).

[8] T.F. Schulze, et al., Phys. Rev. B 83(16), 165314 (2011).

[9] W. Jackson, et al., Phys. Rev. B 31(8) 5187 (1985).

[10] J. Tauc, Mater. Res. Bull. 3, 37 (1968).

[11] Jacques I. Pankove, in Optical Processes in Semiconductors Dover edition (Dover Publications, New York, 1975).

[12] P. Yu and M. Cardona, in Fundamentals of Semiconductors: Physics and Materials Properties Third, Revised and Enlarged Edition (Springer, Berlin, 2005).

[13] F. Urbach Phys. Rev. 51(5), 1324 (1953).

[14] J. J. Thevaril et al., J. Appl. Phys. 120(13), 135706 (2016).

[15] S. K. O'Leary et al., J. Appl. Phys. 92(8), 4276 (2002).

[16] T. Skettrup, Phys. Rev. B 18(6) (1978).

[17] S. John et al., Phys. Rev. Lett. 57(14), 1777 (1986).

[18] S. K. O'Leary et al., Phys. Rev. B 52(11), 7795 (1995).

[19] S. K. O'Leary et al., Phys. Rev. B 51(7), 4143 (1995).

[20] N. F. Mott and E. A. Davis, in Electronic Processes in Noncrystalline Materials (Oxford University Press, Oxford, 1979).

[21] P. B. Allen, in Conceptual Foundations of Materials: A Standard Model for Ground- and Excited-State Properties, Chapter 6, (Elsevier, B.V., 2006).

[22] L. L. Moseley et al., Am. J. Phys. 46(6), 676-677 (1978).

[23] B. Ullrich et al., Jap. J. Appl. Phys. 30(7B), 1285 (1991). 
[24] B. Ullrich et al., J. Appl. Phys. 93(4), 1914 (2003).

[25] A. S. Ferlauto et al., J. Appl. Phys. 92(5), 2424-2436 (2002).

[26] C. M. Soukoulis et al., Phys. Rev. Lett. 53(6), 616 (1984).

[27] S. Abe and Y. Toyozawa, J. Phys. Soc. Jpn. 50, 2185 (1981).

[28] D. J. Dunstan, J. Phys. C: Sol. Stat. Phys. 16(17), L567 (1983).

[29] D. J. Dunstan, J. Phys. C: Sol. Stat. Phys. 18(28), 5429 (1985).

[30] L. Jiao et al., Appl. Phys. Lett. 72(9), 1057 (1998).

[31] R. A. Street, in Hydrogenated Amorphous Silicon (Cambridge University Press, Cambridge, 1992).

[32] J. Singh and K. Shimakawa, in Advances in Amorphous Semiconductors (Taylor \& Francis, London, 2003).

[33] H. C. Casey et al., J. Appl. Phys. 46(1), 250 (1975).

[34] D. M. G. Leite et al., J . Phys.: Condens. Matter. 20(5), 055001 (2008).

[35] R. E. Viturro et al., Philos. Mag. B 53(2), 93 (1986).

[36] N. Preissler et al., Phys. Stat. Sol. A 213(7), 1697 (2016).

[37] E. Fred Schubert, in Doping in III-V Semiconductors (Cambridge University Press, Cambridge, 1993).

[38] L. R. Tessler and I. Solomon, Phys. Rev. B 52(15), 10962 (1995).

[39] M. Liebhaber et al., Appl. Phys. Lett. 106(3), 031601 (2015).

[40] B. Ullrich et al., Semicond. Sci. Technol. 22(10) 1174 (2007). 